\title{
Determining the density dependence of immigration and emigration of benthic stream invertebrates: theoretical considerations ${ }^{1}$
}

\author{
William Y. B. Chang \& Daniel W. Sell \\ Great Lakes Research Division, The University of Michigan, Ann Arbor, MI 48109, U.S.A.
}

Keywords: density dependence, immigration, emigration, benthic stream, invertebrates

\begin{abstract}
Simple mathematical models are formulated to describe density independent and density dependent dispersal. These models clarify hypotheses of density dependence and may be manipulated easily to suit particular applications. The models demonstrate that the initial composition of a species aggregate must be controlled before valid conclusions can be drawn about the density dependency of the aggregate's dispersal. Stochastic models of emigration are derived to assess the power of particular experimental designs and statistical techniques to discriminate a known form of density dependent emigration.
\end{abstract}

\section{Introduction}

The density dependence of animal dispersal has drawn considerable attention in the ecological literature. There are two implications of density dependent population attributes: 1) biologically important interactions between individuals in the population influence the attribute, and 2) patterns of population dynamics may be recognized and in some way generalized to other populations. For stream benthic invertebrates, a distinctive mode of migration is drift (Waters, 1972), but movement on or within the substrate is also important (Hughes, 1970; Hultin, 1971; Williams \& Hynes, 1976; Williams, 1977). Many studies have attempted to assess the relationship between benthic density and dispersal rates (Bovbjerg, 1964; Waters, 1965; Dimond, 1967; Pearson \& Franklin, 1968; Hughes, 1970; Lehmkuhl \& Anderson, 1972; Pearson \& Kramer, 1972; Reisen \& Prins, 1972; Madsen, 1976; Walton et al., 1977). To further determine if migration rates for a particular species or group of species are dependent on density, a few studies have been

\footnotetext{
${ }^{1}$ Contribution No. 369, Great Lakes Research Division, University of Michigan
}

conducted with known initial populations in experimental pools (Corkum, 1978), in artificial streams (Hildebrand, 1974) or in natural waters (Peckarsky, 1979). Studies that have gone beyond descriptive treatments to perform tests have done so on the basis of several well stated hypotheses of density dependence (Reisen \& Prins, 1972; Hildebrand, 1974; Walton et al., 1977; Corkum, 1978). However, shortcomings in 'verbal' models of density dependence have led to vague or inappropriate analyses (Sell, 1981).

In this paper, we present mathematical models for density dependent and independent migration, and examine criteria needed for establishing the role of density in experimental populations. These models complement and extend Sheldon's (1977) mathematical formulations for colonization. We also use stochastic simulations for evaluating experimental designs and consider practical aspects in the application of these models.

\section{Density independent emigration and immigration}

A model which illustrates conditions required for density independent emigration and immigration is: 
$\mathrm{N}(\mathrm{t})=\mathrm{N}(\mathrm{t}-1)(1-\alpha)+\mathrm{B}$

where $N(t)$ is the density of a species on a site at time $t$, measured in discrete intervals, $\alpha$ is the proportion of invertebrates that leave the site in the interval $(t-1)$ to $t$, and $B$ is the number of immigrants moving onto the site in the same interval. The net change (NC) in density over the interval is:

$\mathrm{NC}=\mathrm{B}-\alpha \mathrm{N}(\mathrm{t}-1)$

where $N C=N(t)-N(t-1)$. For a time interval, $N C$ will be a linear function of $N(t-1)$ having a slope of $-\alpha$ and intercept $B$, given that $B$ and $\alpha$ remain constant. $\mathrm{NC}$ is plotted against a range of initial densities for selected values of $\mathrm{B}$ and $\alpha$ in Fig. 1. The same model would apply if $B$ and $\alpha$ were characteristics of an entire fauna rather than a particular species as long as inter- and intraspecific interactions do not occur. Note that dispersal is density independent because the tendencies of animals to immigrate and emigrate, $B$ and $\alpha$ respectively, are not dependent upon treatment values of density $\mathrm{N}(\mathrm{t}-1)$. The distinction between density independent immigration and emigration processes is important. We do not adopt Peckarsky's (1981) formulation of density independent immigration, in which immigration is assumed to be a constant proportion of animals already on the site.

Sheldon (1977) describes a continuous time model for density independent immigration and emigration that is analogous to the discrete time model presented above. Ulfstrand (1968) and Corkum (1978) both appear to use continuous time

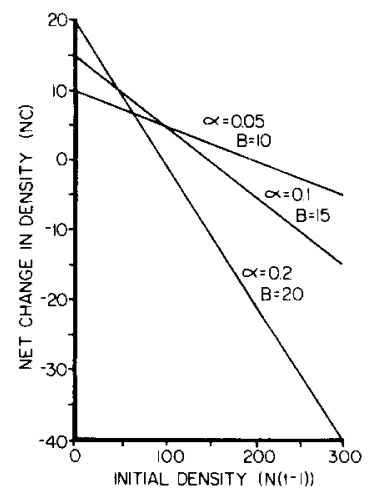

Fig. 1. Net change in numbers versus initial density for density independent emigration and immigration. Parameter values for $\alpha$ and $B$ are shown. models to describe density independent processes but neither author specifies a model in detail. The purpose of using discrete time models here is to allow simple stochastic simulations to be performed.

\section{Density dependent emigration}

Although the concept of density independent immigration allows a biologically meaningful interpretation, the notion of density dependent immigration may not be appropriate in describing invertebrate colonization in streams. Density dependent immigration implies that an individual can sense the density of animals already on the site before it moves onto the site. Because there is little evidence at present, other than Peckarsky (1980), that stream invertebrates can sense the presence of neighbors at much of a distance, immigration will not be considered to be density dependent in subsequent models.

Models with density dependent emigration can be obtained by allowing $\alpha$ to vary with density $N(t-1)$. A simple form of density dependence can be specified by high tendencies to leave a site under crowded conditions and low tendencies to leave under uncrowded conditions. One such formulation is

$\alpha=N(t-1) A^{-1} \quad$ for $0 \leqslant N(t-1) \leqslant A$

where $\mathrm{A}$ is a positive constant. NC may then be described:

$\mathrm{NC}=\mathrm{B}-\{\mathrm{N}(\mathrm{t}-1)\}^{2} \mathrm{~A}^{-1}$

Figure 2 depicts the shapes and positions of the curves to be expected by varying parameters $A$ and B. Even though this formulation is a particular case of density dependence, any type of density dependent emigration would produce some form of curvature in the NC versus initial density response line.

\section{Model application}

An example using data from a hypothetical drift experiment is included to show a practical application of these models. The experiment used four 


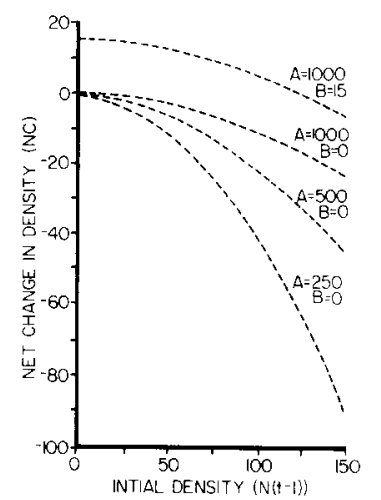

Fig. 2. Net change in numbers versus initial density for density dependent emigration. Parameter values for $A$ and $B$ are shown.

density treatments, each with three replicates. The initial numbers of animals present in a replicate under the four density conditions were 10,20,40 and 80 . Net change (NC) for each level of initial density was measured and is shown in Table 1. No immigration $(\mathbf{B}=\mathbf{0})$ was present during the experiment.

The steps for applying the proposed model are: 1) fit data with a 2 nd order polynomial regression using NC as dependent variable and the initial den-

Table 1. An example is shown using data from a hypothetical drift experiment to show a practical use of the models presented. The experiment used four density treatments, each with three replicates. Net change (NC) in number of animals for each level of initial density is shown, followed by a polynomial regression analysis of the data. No immigration ( $B=0$ ) was noted during the experiment. $B$ is the number of immigrants moving onto the site, $\alpha$ is the proportion of invertebrates that leave the site, and $\mathrm{A}$ is the maximum population that the system is able to support. ns - not significantly different from zero. ${ }^{* * *}$ - significant at 0.001 .

\begin{tabular}{lcccc}
\hline & \multicolumn{4}{c}{ Initial Density (\# animals unit ${ }^{-1}$ area) } \\
Replicate & 10 & 20 & 40 & 80 \\
\hline 1 & 0 & -4 & -12 & -50 \\
2 & -1 & -6 & -10 & -46 \\
3 & -2 & -5 & -9 & -55 \\
\hline
\end{tabular}

\begin{tabular}{lclll}
\hline & \multicolumn{4}{c}{ Polynomial Regression Analysis } \\
& Coefficient & Signif. & $\begin{array}{l}\text { Adjusted } \\
\text { Coefficient }\end{array}$ & Signif. \\
\hline B & 1.188 & (ns) & 0.0 & \\
$\alpha$ & -0.055 & (ns) & 0.0 & \\
A ${ }^{1}$ & 0.0078 & $(* * *)$ & 0.0073 & $(* * *)$ \\
\hline
\end{tabular}

sity as the independent variable; 2) select those coefficients that are not significantly different from zero and set them equal to zero; and 3) rerun the statistical analysis. Following these steps, the result of the application can be obtained as shown in the column of the adjusted coefficients (Table 1), which are the estimates for the parameters of the models presented. Using these coefficients, we have determined that the emigration in this experiment is a form of density dependence (as shown in equation 4 ), and confirmed that immigration onto the site is negligible. The maximum population that the system is able to support can be estimated by inverting the coefficients for the 2 nd order term of the equation; the result in this case is 137 .

\section{Statistical methods for distinguishing density de- pendent and density independent emigration}

The criterion to separate density independence from density dependence of emigration in these simple models may be stated in either of two ways: 1) whether NC versus initial density is a linear response, or 2) whether the proportion of animals leaving the site remains constant at all densities. Several statistical techniques have been used to examine these criteria, but the use of correlation analyses to make inferences about the density dependence of population regulation has been soundly criticized by Eberhardt (1970). Because correlations do not provide a means to distinguish linear and non-linear models, simple correlations of number emigrating or drifting versus density do not allow an interpretation of density responses (Madsen, 1976).

Regression techniques have also been used to examine statistically the density dependence of emigration or drift (Reisen \& Prins, 1972; Hildebrand, 1974; Walton et al., 1977; Sheldon, 1977; Corkum, 1978). Typically, the number emigrating or drifting has been regressed against initial density. In this analysis, a good fit for a linear regression provides evidence for density independence while good fits for non-linear models indicate responses are density dependent. A problem arises, however, when both linear and non-linear models acceptably fit a data set. This is exemplified by the work of Walton et al.. (1977), who show a good fit between linear, exponential, and power functions and drift versus 
initial density data. Thus, further analysis was required to choose between the models.

A meaningful criterion for choosing between linear and non-linear regression models is to employ a stepwise polynomial regression analysis. If higher order terms do not show a significant additional fraction of the variation, then the null hypothesis of a linear (density independent) model is accepted.

A second approach allows a more general test of whether animals have different tendencies to emigrate under different density conditions. Fleiss (1973) describes a chi square analysis for differences in proportions among quantitatively ordered samples. This three step procedure tests the null hypotheses: 1) the proportions are the same for all samples, 2) the proportions show no overall trend to increase or decrease among the quantitatively ordered samples, and 3 ) the tendency of proportions to increase or decrease among the quantitatively ordered samples is linear. If proportions of animals emigrating do not differ significantly between samples then the null hypothesis of density independent emigration is accepted.

\section{Models for species aggregates}

Species have been found to differ in tendencies to immigrate and emigrate (Williams \& Hynes, 1976; Corkum, 1978; Peckarsky, 1979) or to appear in the drift (Waters, 1972). Even within a species, particular life stages may be more inclined or susceptible to drift. Nevertheless, recent studies (Hildebrand, 1974; Peckarsky, 1979) have examined species aggregates because of constraints of field work, for taxonomic considerations, or because an overall description of a benthic community was desired.

To examine the possible response of NC to $\mathrm{N}(\mathrm{t}-1)$ for a species aggregate, consider an aggregate composed of the three species described by density independent relations in Fig. 1. If the assumption is made that species in an aggregate act independently of one another, the aggregate NC response to density can be calculated as the sum of the individual species $\mathrm{NC}$ responses to their own densities. At a zero initial density, the predicted aggregate $\mathrm{NC}$ is $\mathbf{4 5}$ for this set of species. In general, the aggregate $\mathrm{Y}$-axis intercept will equal the sum of the $\mathrm{Y}$-axis intercepts of the individual species lines. At all other initial aggregate densities NC may vary depending upon the initial composition of the aggregate. If the initial composition of the aggregate is taken to be entirely individuals of Species 1, then the aggregate $\mathrm{NC}$ response would correspond to the Species 1 response in Fig. 1 translated vertically (Fig. 3, line A). Likewise, if the initial composition of the aggregate is taken to be only individuals of Species 3 , then the aggregate $\mathrm{NC}$ response would be translated as line D in Fig. 3. Because Species 1 and Species 3 have the extreme tendencies to emigrate (extreme values of $\alpha$ ), all possible aggregate $\mathrm{NC}$ responses to $N(t-1)$ will be bounded by these two lines. If the initial relative abundances of the three species vary at different initial aggregate densities, then non-linear $\mathrm{NC}$ responses for the aggregate may be obtained (Fig. 3, line C), even though each species acts in a density independent fashion and all species act independently of one another. The relative abundance of all species in the experimental treatments must be held constant at all initial densities before a linear response of the aggregate $\mathrm{NC}$ to $N(t-1)$ will be obtained (Fig. 3, lines A, B \& D). In general, the greater the difference between the extreme values of $\alpha$ for a set of species, the wider the bounds become for the aggregate response. Also, as initial density increases, larger variations in the magnitude of $\mathrm{NC}$ are possible.

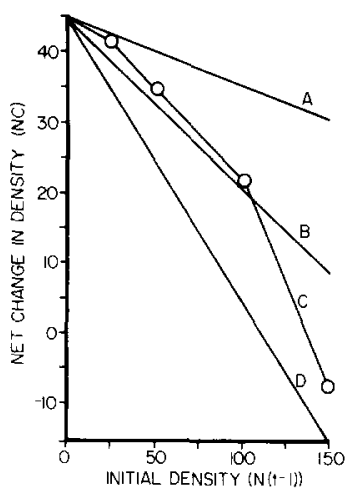

Fig. 3. Net change in numbers versus initial density for an aggregate of three species, each of which acts with density independent emigration and immigration. Parameter values of $\alpha$ and $B$ for the three species are: Species 1, 0.05 and 10; Species 2, 0.1 and 15 ; Species 3,0.2 and 20. Lines $A$ and $D$ represent the bounds for the $\mathrm{NC}$ response line of the aggregate. For line $\mathrm{C}$, the initial relative abundances of the three species varied at five experimental densities. For line B, the proportions of the three species remain constant $(0.2,0.5$, and 0.3 respectively for Species 1,2 and 3$)$ at all initial densities. 


\section{Stochastic models of emigration}

The above models are deterministic in that variations in the predicted response have not been considered. In this section we will consider simulations of random emigration, which are made by considering the probability that each animal on the site will stay or leave in each discrete time interval, with each animal having an $\alpha$ probability of leaving. The probability that $\mathrm{X}$ of the $\mathrm{N}(\mathrm{t}-\mathrm{l})$ animals will remain on the site in the interval $(t-1)$ to $t$ may then be described by the binomial probabilities:

$\operatorname{Pr}\{\mathrm{N}(\mathrm{t})=\mathrm{X} \mid \mathrm{N}(\mathrm{t}-1), \alpha\}=$

$\{\mathrm{N}(\mathrm{t}-1)\}(1-\alpha)^{\mathrm{X}} \alpha^{\mathrm{N}(\mathrm{t}-1)-\mathrm{X}}$

The assumption of this model is that for any density treatment all animals have identical tendencies (probabilities) to emigrate.

Although use of binomial probabilities allows an intuitive interpretation of emigration, use of other probability functions may be justified on empirical grounds. For example, the number of animals remaining on the site may be described by Poisson probabilities:

$\operatorname{Pr}\{N(t)=X \mid N(t-1), \alpha\}=\left(\lambda^{X} \mathrm{e}^{-\lambda}\right) / X !$

where $\lambda=\mathrm{N}(\mathrm{t}-1) \alpha$.

Stochastic simulations yield NC versus $N(t-1)$ responses which may be described as a probability envelope around a deterministic response line. Note that these stochastic formulations are applied to either the density independent or density dependent models with discrete time formulations.

The power of statistical analyses to distinguish density dependent or independent emigration is evaluated using stochastic simulations. The example below shows the results of an evaluation for the same experimental design used in the example of Table 1. Ten experimental runs were made for each of 3 parameter values of A (maximum animal density). The results of each experimental run were analyzed by stepwise polynomial regression and by the chi square analyses for differences in proportions.

The results of simulations using binomial and Poisson probabilities are shown in Table 2. The parameter value $A=1000$ provides the least sensi- tive response to density over the initial density range of $10-80$ animals unit ${ }^{-1}$ area, and $A=250$ provides the most dramatic response. The proportion of experimental runs which correctly distinguished (at the 0.05 confidence level) the underlying density dependent model is given for the first two tests of the chi square analysis and for the polynomial regression analysis. All three statistical tests performed well in simulations employing the most dramatic density response. The greater variability introduced by Poisson probabilities masks the underlying model more effectively than do binomial probabilities.

\section{Discussion}

Two essential conditions are suggested in this study for testing hypotheses regarding the effects of density on emigration and immigration of stream invertebrates in in situ experiments. First, the relative abundances of species must not vary with initial density. Second, non-linear and linear responses of NC or number versus initial density must be distinguishable. If these conditions are met, then non-linear changes in NC would indicate that dispersal is density dependent, the probability of immigration or emigration varying with initial treatment density. Because the calculation of immigration or emigration rates for a standardized set of species with particular relative abundances has little intuitive meaning, migration measures of aggregates have limited usefulness. This conclusion reinforces the importance of investigations at the species level.

Field studies of density dependence should also consider the fact that in natural populations, density must reach a level where interactions can occur before density dependent emigration is possible. Below this lower critical density, immigration and emigration are density independent. On the other hand, all animals may be expected to sense and react to others under crowded conditions. Even if a taxon's tendency to emigrate and immigrate is independent of density at low densities, density dependent responses may be expected above an upper critical density. Besides the range of densities examined, other factors such as sediment type and current velocity may also complicate the analysis (Walton et al., 1977).

Immigration of benthic invertebrates to a site 
Table 2. Results of statistical analyses of emigration data generated from stochastic simulations. Significance levels are given. ns $=$ not significant at $p=0.10$. The parameter $A$ specifies the degree of density dependence. The fraction of runs (FR) for which the underlying density dependent model was distinguished at $\mathrm{p}=0.05$ is given. POLY denotes stepwise polynomial regression, $\mathrm{CHI} 1 \mathrm{the}$ first test of the chi square analysis, and $\mathrm{CHI} 2$ the second test.

\begin{tabular}{|c|c|c|c|c|c|c|c|c|c|}
\hline \multirow[b]{3}{*}{ Run } & \multicolumn{9}{|c|}{ Binomial stochastic model } \\
\hline & \multicolumn{3}{|c|}{$A=1000$} & \multicolumn{3}{|c|}{$\mathrm{A}=500$} & \multicolumn{3}{|c|}{$A=250$} \\
\hline & POLY & $\mathrm{CHI} 1$ & $\mathrm{CHI} 2$ & POLY & $\mathrm{CHI} I$ & $\mathrm{CH} 12$ & POLY & CHI 1 & $\mathrm{CHI} 2$ \\
\hline 1 & ns & ns & .10 & .05 & .05 & .01 & .0005 & .0005 & .0005 \\
\hline 2 & .01 & .05 & .005 & .025 & .005 & .0005 & .0005 & .0005 & .0005 \\
\hline 3 & .005 & .01 & .005 & .001 & .0005 & .0005 & .001 & .0005 & .0005 \\
\hline 4 & ns & ns & .10 & .005 & .0005 & .0005 & .001 & .0005 & .0005 \\
\hline 5 & .05 & .005 & .0005 & .001 & .0005 & .0005 & .0005 & .0005 & .0005 \\
\hline 6 & .005 & ns & .05 & .005 & .025 & .005 & .001 & .0005 & .0005 \\
\hline 7 & .005 & .10 & .025 & .005 & .005 & .0005 & .001 & .0005 & .0005 \\
\hline 8 & .0005 & .0005 & .0005 & .10 & .01 & .005 & .0005 & .0005 & .0005 \\
\hline 9 & .001 & .10 & .10 & .01 & .005 & .0005 & .0005 & .0005 & .0005 \\
\hline 10 & .025 & ns & ns & .05 & .10 & .01 & .0005 & .0005 & .0005 \\
\hline \multirow[t]{2}{*}{ FR } & $8 / 10$ & $4 / 10$ & $6 / 10$ & $9 / 10$ & $9 / 10$ & $10 / 10$ & $10 / 10$ & $10 / 10$ & $10 / 10$ \\
\hline & \multicolumn{9}{|c|}{ Poisson stochastic model } \\
\hline Run & POLY & $\mathrm{CHI} \mathrm{I}$ & $\mathrm{CHI} 2$ & POLY & $\mathrm{CHI} 1$ & CHI 2 & POLY & CHI I & CHI 2 \\
\hline 1 & ns & .10 & .05 & .005 & .10 & .01 & .025 & .0005 & .0005 \\
\hline 2 & .10 & $\mathrm{~ns}$ & .05 & $\mathrm{~ns}$ & ns & .10 & .01 & .0005 & .0005 \\
\hline 3 & .10 & ns & $\mathrm{ns}$ & .05 & .005 & .0005 & .0005 & .0005 & .0005 \\
\hline 4 & .025 & .005 & .0005 & $\mathrm{~ns}$ & ns & .025 & .0005 & .0005 & .0005 \\
\hline 5 & .10 & $\mathrm{~ns}$ & .10 & .10 & .01 & .0005 & .0005 & .0005 & .0005 \\
\hline 6 & .01 & .01 & .005 & .001 & .005 & .0005 & .0005 & .0005 & .0005 \\
\hline 7 & .0005 & .005 & .0005 & .10 & .10 & .01 & .005 & .0005 & .0005 \\
\hline 8 & ns & $\mathrm{ns}$ & .05 & .0005 & .05 & .01 & .005 & .0005 & .0005 \\
\hline 9 & .005 & $\mathrm{~ns}$ & .05 & .01 & .005 & .0005 & .0005 & .0005 & .0005 \\
\hline 10 & .005 & .025 & .005 & .005 & .0005 & .0005 & .025 & .0005 & .0005 \\
\hline FR & $5 / 10$ & $4 / 10$ & $8 / 10$ & $6 / 10$ & $6 / 10$ & $9 / 10$ & $10 / 10$ & $10 / 10$ & $10 / 10$ \\
\hline
\end{tabular}

may be independent of the number of individuals already existing at that site. This should hold for unidirectional flowing systems such as streams and rivers, and assumes that those immigrating cannot foretell the existing density. However, immigration processes which result in a selective number of organisms colonizing a site may be conceptualized differently. Walton (1978) describes substrate attachment from drift as a two step process - settling onto a site in an apparently unselective manner and a selective step involving rapid reentry into the drift. A density dependent analogue to Walton's (1978) 'trial' attachment process could be modeled by density independent immigration and a rapid density dependent emigration.

The chief requirement for more detailed and meaningful analyses of immigration and emigration within streams is the ability to monitor the movements of individuals. As marking methods or photographic techniques develop to track individuals (Wiley \& Kohler, 1981), immigration and emigration rates may be independently estimated. Direct observation of invertebrate behavior (Peckarsky, 1980) may allow mechanisms of biological interactions to be more clearly defined.

\section{Acknowledgements}

We thank Drs. Steven Obrebski, Malcolm Butler, John Lehman, Vincent Resh, David White and Mike Wiley for helpful comments and criticisms. 


\section{References}

Bovbjerg, R. V., 1964. Dispersal of aquatic animals relative to density. Verh. int. Ver. Limnol. 15: 879-884.

Corkum, L. D., 1978. The influence of density and behavioural type on the active entry of two mayfly species (Ephemeroptera) into the water column. Can. J. Zool. 56: 1201-1206.

Dimond, J. B., 1967. Evidence that drift of stream benthos is density related. Ecology 48: 855-857.

Eberhardt, L. L., 1970. Correlation, regression, and density dependence. Ecology 51: 306-310.

Fleiss, J. L., 1973. Statistical methods for rates and proportions. John Wiley \& Sons, Inc., N.Y., 321 pp.

Hildebrand, S. C., 1974. The relation of drift to benthos density and food level in an artificial stream. Limnol. Oceanogr. 19: 951-957.

Hughes, D. A., 1970. Some factors affecting drift and upstream movements of Gammarus pulex. Ecology 51: 301-305.

Hultin, L.., 1971. Upstream movements of Gammarus pulex pulex (Amphipoda) in a South Swedish stream. Oikos 22: 329-347.

Lehmkuhl, D. M. \& N. H. Anderson, 1972. Microdistribution and density as factors affecting the downstream drift of mayflies. Ecology 53: 661-667.

Madsen, B. L., 1976. Population dynamics of Brachyptera risi nymphs (Plecoptera). Oikos 27: 281-287.

Pearson, W. D. \& D. R. Franklin, 1968. Some factors affecting drift rates of Baetis and Simuliidae in a large river. Ecology 49: $75-81$.

Pearson, W. D. \& R. H. Kramer, 1972. Drift and production of two aquatic insects in a mountain stream. Ecol. Monogr. 42: 365-385

Peckarsky, B. L., 1979. Biological interactions as determinants of distributions of benthic invertebrates within the substrate of stony streams. Limnol. Oceanogr. 24: 59-68.
Peckarsky, B. L., 1980. Predator-prey interactions between stoneflies and mayflies: behavioral observations. Ecology 61: 932-943.

Peckarsky, B. L., 1981. Reply to comment by Sell. Limnol. Oceanogr. 26: 982-987.

Reisen, W. K.\& R. Prins, 1972. Some ecological relationships of the invertebrate drift in Praters Creek, Pickens County, South Carolina. Ecology 53: 876-884.

Sell, D. W., 1981. Comment on the analysis of biological interactions of stream invertebrates. Limnol. Oceanogr. 26: 981-982.

Sheldon, A. L., 1977. Colonization curves: application to stream insects on semi-natural substrates. Oikos 28: 256-261.

Ulfstrand, S., 1968. Benthic animal communities in Lapland streams. Oikos Suppl. 10: 1-120.

Walton, O. E. Jr., S. R. Reice \& R. W. Andrews, 1977. The effects of density, sediment particle size and velocity on drift of Acroneuria abnormis (Plecoptera). Oikos 28: 291-298.

Walton, O. E. Jr, 1978. Substrate attachment by drifting aquatic insect larvae. Ecology 59: 1023-1030.

Waters, T.F., 1965. Interpretation of invertebrate drift in streams. Ecology 46: $327-334$.

Waters, T. F., 1972. The drift of stream insects. Annu. Rev. Entomol. 17: 253-272.

Wiley, M. \& S. Kohler, 1981. An assessment of biological interactions in an epilithic stream community using time lapse cinematography. Hydrobiologia 78: 183-188.

Williams, D. D., 1977. Movements of benthos during the recolonization of temporary streams. Oikos 29: 306-312.

Williams, D. D. \& H. B. N. Hynes, 1976. The recolonization of stream benthos. Oikos 27: 265-272.

Received 4 July 1982; in revised form 15 March 1983; accepted 13 April 1983 